\title{
Thermal expansion feedback for wave-front shaping
}

\author{
Omer Tzang, " Eyal Niv, Antonio M. Caravaca-AguirRe, And Rafael \\ PiEstun \\ Department of Electrical, Computer, and Energy Engineering, University of Colorado, Boulder, \\ Colorado 80309, USA \\ *omer.tzang@colorado.edu
}

\begin{abstract}
Focusing inside scattering media is a challenging task with a variety of applications in biomedicine. State of the art methods mostly require invasive feedback inside or behind the sample, limiting practical use. We present a technique for dynamic control and focusing inside scattering media that combines two powerful methods: optical coherence tomography (OCT) and wave-front shaping (WFS). We use OCT as a non-invasive feedback for WFS optimization of a separate, penetrating laser. Energy absorbed in the sample, creates thermal expansions that are used for the feedback mechanism. By maximizing thermal deformations within a selected focal region, we demonstrate enhanced focusing of light through scattering media beyond the ballistic regime and within the penetration range of OCT.
\end{abstract}

(C) 2017 Optical Society of America

OCIS codes: (110.0113) Imaging through turbid media; (110.7348) Wavefront encoding; (110.4500) Optical coherence tomography.

\section{References and links}

1. I. M. Vellekoop, and A. P. Mosk, "Focusing coherent light through opaque strongly scattering media," Opt. Lett. 32(16), 2309-2311 (2007).

2. A. P. Mosk, A. Lagendijk, G. Lerosey, and M. Fink, "Controlling waves in space and time for imaging and focusing in complex media," Nat. Photonics 6(5), 283-292 (2012).

3. I. M. Vellekoop, A. Lagendijk, and A. P. Mosk, "Exploiting disorder for perfect focusing," Nat. Photonics 4, 320-322 (2010).

4. O. Katz, E. Small, and Y. Silberberg, "Looking around corners and through thin turbid layers in real time with scattered incoherent light," Nat. Photonics 6(8), 549-553 (2012).

5. I. M. Vellekoop, "Feedback-based wavefront shaping," Opt. Express 23(9), 12189-12206 (2015)

6. G. Lerosey, J. de Rosny, A. Tourin, and M. Fink, "Focusing beyond the diffraction limit with far-field time reversal," Science 315(5815), 1120-1122 (2007).

7. S. Popoff, G. Lerosey, M. Fink, A. C. Boccara, and S. Gigan, "Image transmission through an opaque material," Nat. Commun. 1(6), 81 (2010).

8. C.-L. Hsieh, Y. Pu, R. Grange, and D. Psaltis, "Digital phase conjugation of second harmonic radiation emitted by nanoparticles in turbid media," Opt. Express 18(12), 12283-12290 (2010).

9. I. M. Vellekoop, M. Cui, and C. Yang, "Digital optical phase conjugation of fluorescence in turbid tissue," Appl. Phys. Lett. 101(8), 081108 (2012).

10. X. Tao, O. Azucena, M. Fu, Y. Zuo, D. C. Chen, and J. Kubby, "Adaptive optics microscopy with direct wavefront sensing using fluorescent protein guide stars,” Opt. Lett. 36(17), 3389-3391 (2011).

11. D. B. Conkey, A. M. Caravaca-Aguirre, J. D. Dove, H. Ju, T. W. Murray, and R. Piestun, "Super-resolution photoacoustic imaging through a scattering wall," Nat. Commun. 6, 9 (2015).

12. F. Kong, R. H. Silverman, L. Liu, P. V. Chitnis, K. K. Lee, and Y. C. Chen, "Photoacoustic-guided convergence of light through optically diffusive media," Opt. Lett. 36(11), 2053-2055 (2011).

13. T. Chaigne, O. Katz, C. Boccara, M. Fink, E. Bossy, and S. Gigan, "Controlling light in scattering media noninvasively using the photoacoustic transmission matrix," Nat. Photonics 8(1), 58-64 (2013).

14. X. Xu, H. Liu, and L. V. Wang, "Time-reversed ultrasonically encoded optical focusing into scattering media," Nat. Photonics 5(3), 154-157 (2011).

15. B. Judkewitz, Y. M. Wang, R. Horstmeyer, A. Mathy, and C. Yang, "Speckle-scale focusing in the diffusive regime with time-reversal of variance-encoded light (TROVE)," Nat. Photonics 7(4), 300-305 (2013).

16. C. Dunsby and P. M. W. French, "Techniques for depth-resolved imaging through turbid media including coherence-gated imaging," J. Phys. D Appl. Phys. 36(14), R207-R227 (2003).

17. D. Huang, E. A. Swanson, C. P. Lin, J. S. Schuman, W. G. Stinson, W. Chang, M. R. Hee, T. Flotte, K. Gregory, C. A. Puliafito, and J. G. Fujimoto, "Optical coherence tomography," Science 254(5035), 1178-1181 (1991). 
18. J. Schmitt, “Optical Coherence Tomography (OCT): A Review,” IEEE J. Sel. Top. Quantum Electron. 5(4), $1205-1215$ (1999).

19. R. Fiolka, K. Si, and M. Cui, "Complex wavefront corrections for deep tissue focusing using low coherence backscattered light,” Opt. Express 20(15), 16532 (2012).

20. J. Jang, J. Lim, H. Yu, H. Choi, J. Ha, J.-H. Park, W.-Y. Oh, W. Jang, S. Lee, and Y. Park, "Complex wavefront shaping for optimal depth-selective focusing in optical coherence tomography," Opt. Express 21(3), 2890-2902 (2013).

21. H. Yu, J. Jang, J. Lim, J.-H. Park, W. Jang, J.-Y. Kim, and Y. Park, "Depth-enhanced 2-D optical coherence tomography using complex wavefront shaping," Opt. Express 22(7), 7514-7523 (2014).

22. T. R. Hillman, Y. Choi, N. Lue, Y. Sung, R. R. Dasari, W. Choi, and Z. Yaqoob, "A reflection-mode configuration for enhanced light delivery through turbidity," SPIE BiOS 8227, 82271T (2012).

23. A. Badon, D. Li, G. Lerosey, A. C. Boccara, M. Fink, and A. Aubry, "Smart optical coherence tomography for ultra-deep imaging through highly scattering media," Sci. Adv. 2, e1600370 (2016).

24. R. J. Zawadzki, S. S. Choi, S. M. Jones, S. S. Oliver, and J. S. Werner, "Adaptive optics-optical coherence tomography: optimizing visualization of microscopic retinal structures in three dimensions," J. Opt. Soc. Am. A 24(5), 1373-1383 (2007).

25. B. Hermann, E. J. Fernández, A. Unterhuber, H. Sattmann, A. F. Fercher, W. Drexler, P. M. Prieto, and P. Artal, "Adaptive-optics ultrahigh-resolution optical coherence tomography," Opt. Lett. 29(18), 2142-2144 (2004).

26. K. Kurokawa, K. Sasaki, S. Makita, M. Yamanari, B. Cense, and Y. Yasuno, "Simultaneous high-resolution retinal imaging and high-penetration choroidal imaging by one-micrometer adaptive optics optical coherence tomography.," Opt. Express 18(8), 8515-8527 (2010).

27. M. Guizar-Sicairos, S. T. Thurman, and J. R. Fienup, "Efficient subpixel image registration algorithms.," Opt. Lett. 33(2), 156-158 (2008).

28. D. B. Conkey, A. N. Brown, A. M. Caravaca-Aguirre, and R. Piestun, "Genetic algorithm optimization for focusing through turbid media in noisy environments," Opt. Express 20(5), 4840-4849 (2012).

29. N. Wadhwa, M. Rubinstein, F. Durand, and W. T. Freeman, "Phase-based video motion processing," ACM Trans. Graph. 32(4), 1 (2013).

30. D. C. Adler, S.-W. Huang, R. Huber, and J. G. Fujimoto, "Photothermal detection of gold nanoparticles using phase-sensitive optical coherence tomography," Opt. Express 16(7), 4376-4393 (2008).

31. R. K. Wang, and A. L. Nuttall, "Phase-sensitive optical coherence tomography imaging of the tissue motion within the organ of Corti at a subnanometer scale: a preliminary study," J. Biomed. Opt. 15(5), 056005 (2010).

32. S. J. Kirkpatrick, R. K. Wang, and D. D. Duncan, "OCT-based elastography for large and small deformations," Opt. Express 14(24), 11585-11597 (2006).

33. R. K. Wang, S. Kirkpatrick, and M. Hinds, "Phase-sensitive optical coherence elastography for mapping tissue microstrains in real time," Appl. Phys. Lett. 90, 88-91 (2007).

34. G. M. E. Akkermans, Mesoscopic Physics of Electrons and Photons (Cambridge University Press, 2006).

35. V. Ntziachristos, "Going deeper than microscopy: the optical imaging frontier in biology," Nat. Methods 7(8), 603-614 (2010).

36. N. Bosschaart, D. J. Faber, T. G. van Leeuwen, and M. C. G. Aalders, "Measurements of wavelength dependent scattering and backscattering coefficients by low-coherence spectroscopy," J. Biomed. Opt. 16(3), 030503 (2011).

37. T. Lister, P. A. Wright, P. H. Chappell, T. Lister, P. A. Wright, and P. H. Chappell, "Optical properties of human skin,” J. Biomed. Opt. 17(9), 0909011 (2012).

38. D. T. D. Van der Zee and M. Essenpreis, "Optical properties of brain tissue," Proc. SPIE 1888, 454-465 (1993).

39. S. L. Jacques, "Optical properties of biological tissues: a review," Phys. Med. Biol. 58(11), R37-R61 (2013).

40. S. A. Telenkov, D. P. Dave, S. Sethuraman, T. Akkin, and T. E. Milner, "Differential phase optical coherence probe for depth-resolved detection of photothermal response in tissue," Phys. Med. Biol. 49(1), 111-119 (2004).

41. J. Kim, J. Oh, and T. E. Milner, "Measurement of optical path length change following pulsed laser irradiation using differential phase optical coherence tomography," J. Biomed. Opt. 11(4), 041122 (2006).

42. G. Guan, R. Reif, Z. Huang, and R. K. Wang, "Depth profiling of photothermal compound concentrations using phase sensitive optical coherence tomography," J. Biomed. Opt. 16(12), 126003 (2011).

43. J. M. Tucker-Schwartz, T. A. Meyer, C. A. Patil, C. L. Duvall, and M. C. Skala, "In vivo photothermal optical coherence tomography of gold nanorod contrast agents," Biomed. Opt. Express 3(11), 2881-2895 (2012).

44. S. Makita and Y. Yasuno, "In vivo photothermal optical coherence tomography for non-invasive imaging of endogenous absorption agents," Biomed. Opt. Express 6(5), 1707-1725 (2015).

\section{Introduction}

Recent developments in the field of wave-front shaping (WFS) have demonstrated control and optical focusing through scattering media [1,2]. Coherent light in such media generates randomly scattered light fields, creating random interference patterns, known as speckles. Speckles can be manipulated by controlling the incident wave-front phase, using modern high resolution spatial light modulators and deformable mirrors. Methods for focusing light through scattering media require an adaptive feedback process or phase conjugation to 
approximate the optical modes in the random media. Recent methods include wave-front optimization [3-5], time reversal [6] and directly measuring and inverting the transmission matrix [7]. These approaches require measurements of transmitted light and therefore are not directly applicable for focusing inside scattering media. Alternative methods for focusing light inside scattering media with non-invasive feedback mechanisms include fluorescent guide-stars [8-10], photoacoustics [11-13] time reversal of ultrasound encoded light [14] and time reversal of variance encoded light [15].

Imaging inside scattering media can be performed, to some extent, by coherence gating [16]. In coherence gating the coherence properties of the source provide a mechanism for filtering scattered light. Changes in the optical path exceeding the coherence length, due to scattering events, suppress the interference of scattered photons with a reference beam, whereas ballistic photons interfere strongly. The most widely used form of coherence gating is optical coherence tomography (OCT) [17], which is capable of imaging at depths of a few millimeters inside tissue. OCT has been developed in multiple modalities, including time and frequency domain OCT, the latter realized by spectral domain and swept source systems [18]. OCT is currently a significant biomedical tool in dermatology and ophthalmology. Infra-red (IR) light is typically used to reduce scattering and absorption, enabling deeper imaging in tissue.

Several methods have demonstrated enhanced depth of field and resolution in coherence gated imaging into scattering media. For instance, WFS OCT introduces an active phase modulation element to the OCT scheme [19-23] in order to extend the imaging depth, signal to noise ratio (SNR), or resolution. Aberration correction of the OCT signal has also been demonstrated [24-26], improving OCT resolution and image quality. In these techniques the optimization seeks to enhance the OCT signal.

In this work we integrate the two techniques, WFS and OCT, in a different way and with a different purpose in mind. We use OCT as feedback for WFS optimization of an overlapping, penetrating laser, and not of the OCT source as previously implemented (see Fig. 1). Therefore, we demonstrate a noninvasive feedback approach that relies on light-induced thermal expansion and deformation inside the media: thermal expansion feedback wave-front shaping (TEF-WFS). Accordingly, absorption of light in the sample creates local heating and thermal expansion that deforms the sample. Deformations are detected in the OCT image and are effectively used to modulate the illumination, control scattered light, and focus it into a specific region of interest (ROI). The objective of the method is to enhance energy deposition inside/through a scattering medium by an independent source at a selected wavelength. Combining a second wave-front-shaped laser beam with an OCT system is a realistic goal that could enhance applications such as photodynamic therapy, which often require photons in the visible range tuned to specific chromophore absorption bands. In addition, the OCT arm enables on-line dynamic monitoring of the sample during all phases of energy deposition as well as precise selection of the focusing target inside complex tissue. 


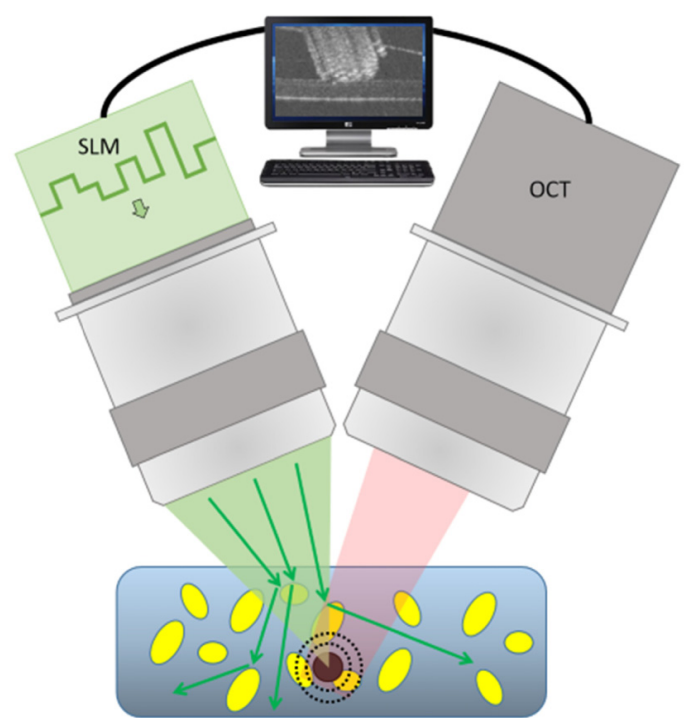

Fig. 1. Principle of TEF-WFS: The sample consists of scattering particles (yellow) and absorbing targets (black). OCT imaging monitors the sample which is illuminated by a coincident, green, penetrating laser. Focusing of the green light on an absorber is attained by WFS using a SLM and by monitoring deformation of absorbing targets in the OCT image.

\section{Thermal expansion feedback: Detecting deformation with OCT}

In order to achieve sensitive detection of deformations inside the scattering sample we implement an OCT-based feedback for wave-front optimization. We rely on elastic, reversible, material thermal expansions that directly translate into shape changes in the OCT image. In order to improve the feedback sensitivity beyond that given by the OCT resolution, we measure translations of selected image features using an interpolated cross-correlation method [27]. The interpolation is based on a frequency-domain zero padding of the Discrete Fourier Transform of the OCT image, forming a x100 representation of the image. For deformation detection, we compute the cross correlation, $C C$, in the Fourier domain and apply the interpolation on the result as follows.

$$
C C\{\boldsymbol{I}, \boldsymbol{T}\}=\operatorname{IDFT}\left\{Z P\left[\operatorname{DFT}(\boldsymbol{I}) \cdot \operatorname{DFT}(\boldsymbol{T})^{*}\right]\right\}
$$

I is a ROI on the OCT image that is captured when the shutter (see Fig. 2) is open and the penetrating laser is heating the sample. $\boldsymbol{T}$ is a reference image template, captured when the shutter is closed and the sample is not heated. Additionally, ZP represents zero padding, DFT and IDFT are the discrete Fourier transform and its inverse respectively.

We use the results of the cross-correlation to find the 2D Euclidean distance that corresponds to the translation (both axial and transverse) of the sample in the OCT image, between a reference (laser off) and a signal (laser on), defined as

$$
\boldsymbol{d}=\sqrt{C C_{x}^{2}+C C_{z}^{2}}
$$

where, $C C_{x}$ and $C C_{z}$ are the offsets of the cross-correlation global peak, defined by $\max (C C\{\boldsymbol{I}, \boldsymbol{T}\})$. The operation is performed on a user defined ROI in the image to allow the selection of a focusing location. Each acquisition is pre-processed using a nonlinear thresholding filter, as depicted in Fig. 3(d), to reduce noise on the signal and improve the cross-correlation result. The deformation in pixel units or its equivalent distance is assigned as the cost value for GA optimization. Due to the large thermal expansions detected in the 
experiment we neglected thermally-induced refractive index changes. We treat all 2D deformations as isotropic, by assigning uniform cost values to all deformations. In anisotropic samples, the cost function could be weighted according to the anisotropic tensor of thermal expansion coefficients, assigning an appropriate cost value (light intensity) to deformations along each axis.

\section{Experimental setup}

The experimental setup is depicted in detail in Fig. 2. The beam to be focused through the scatterer originates from a $1 \mathrm{~W}, 514 \mathrm{~nm}, \mathrm{CW}$ Argon Ion laser (Spectra physics Innova 300), focused by a long working distance objective (x10, 0.15NA) and modulated by an external, mechanical, shutter (UNIBLITZ VMM-D1). The laser power that reaches the sample was up to $500 \mathrm{~mW}$. The modulation (on/off) was performed at $\sim 0.2 \mathrm{~Hz}$, limited by the OCT device acquisition rate of $\sim 5 \mathrm{sec}$ per image. The laser light passes through a standard diffuser (Thorlabs DG10-1500) that serves as a configurable scatterer for each experiment. The distance between the diffuser and the sample was $\sim 8 \mathrm{~mm}$. Wave-front shaping is preformed using a Liquid-Crystal Spatial Light Modulator (LC-SLM) (Holoeye HEO1080p), divided into 144 independent macro-pixels with phase that varies between 0 and $2 \pi$. For feedback, the system includes a commercial OCT (Thorlabs Spectral Radar OCP930SR), comprised of a $930 \mathrm{~nm}$ super luminescent diode (SLD) with a $100 \mathrm{~nm}$ bandwidth and a spectrometer with a $0.14 \mathrm{~nm}$ resolution. The OCT's axial resolution is $6.2 \mu \mathrm{m}$, the maximal imaging depth is $1.6 \mathrm{~mm}$ with a spatial resolution of roughly $10 \mu \mathrm{m}$, due to the low NA lens in the device. The OCT and laser beams overlap at an angle of $80^{\circ}$. A backside camera (Point Grey Research CMLN-13S2C-CS) images the speckle patterns. The shutter, LC-SLM and OCT acquisition are controlled by tailored MATLAB software. A proof-of-concept sample consists of a black polyester sewing thread with a diameter of $200 \mu \mathrm{m}$, fixed on a microscope slide with an external layer of adhesive tape. We selected the black polyester absorbing sample due to its relatively large linear thermal expansion coefficient, $\alpha=123.5 \times 10^{-6}\left[\frac{\mathrm{m}}{\mathrm{mK}}\right]$. The adhesive tape (Scotch@), made of Cellulose Acetate is characterized by low absorption, high scattering and a matching thermal expansion coefficient, $\alpha=130 \times 10^{-6}\left[\frac{\mathrm{m}}{\mathrm{mK}}\right]$. 


\section{Optics EXPRESS}

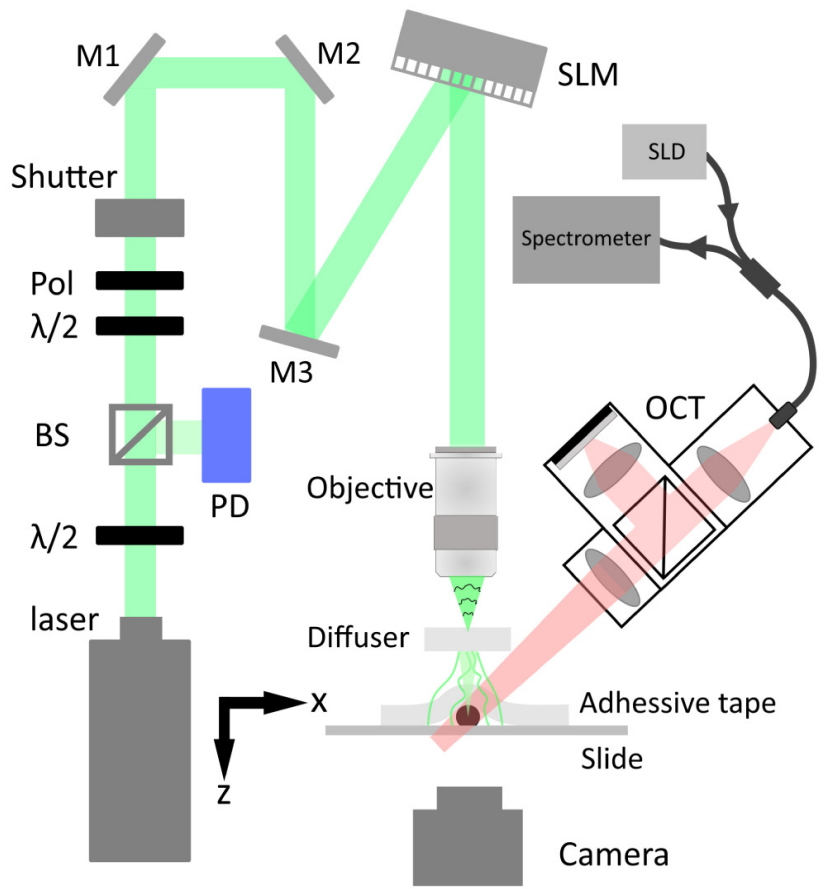

Fig. 2. Experimental setup for TEF-WFS. The $514 \mathrm{~nm} \mathrm{CW}$ laser source is monitored by the Photodiode (PD), modulated by a mechanical shutter and a SLM, and is focused through a diffuser on the sample. A commercial spectral domain OCT monitors the sample over a region overlapping with the desired focal area of the laser.

\section{Results}

Figure 3(a) depicts the characterization of the thermal deformation of the black polyester sewing thread sample as a function of laser power. Figure 3(b) depicts a preliminary characterization that defines the noise floor, and is useful for studying the SNR of the method. In this preliminary step, a series of random patterns followed by a series of constant patterns were projected on the SLM. For each pattern, the deformations of the sample were measured and compared. The higher variance in the deformation, caused by the random patterns (signal), in comparison to the variance of deformation with constant pattern (noise), is indicative of changes in the speckle field with wave-front shaping. Figure 3(c) depicts the OCT gray-scale image of the sample. Figure 3(d) depicts the image after thresholding and a user selected ROI. The ROI includes the low absorption tape that serves as a deformation amplifier for focusing light on the thread. 

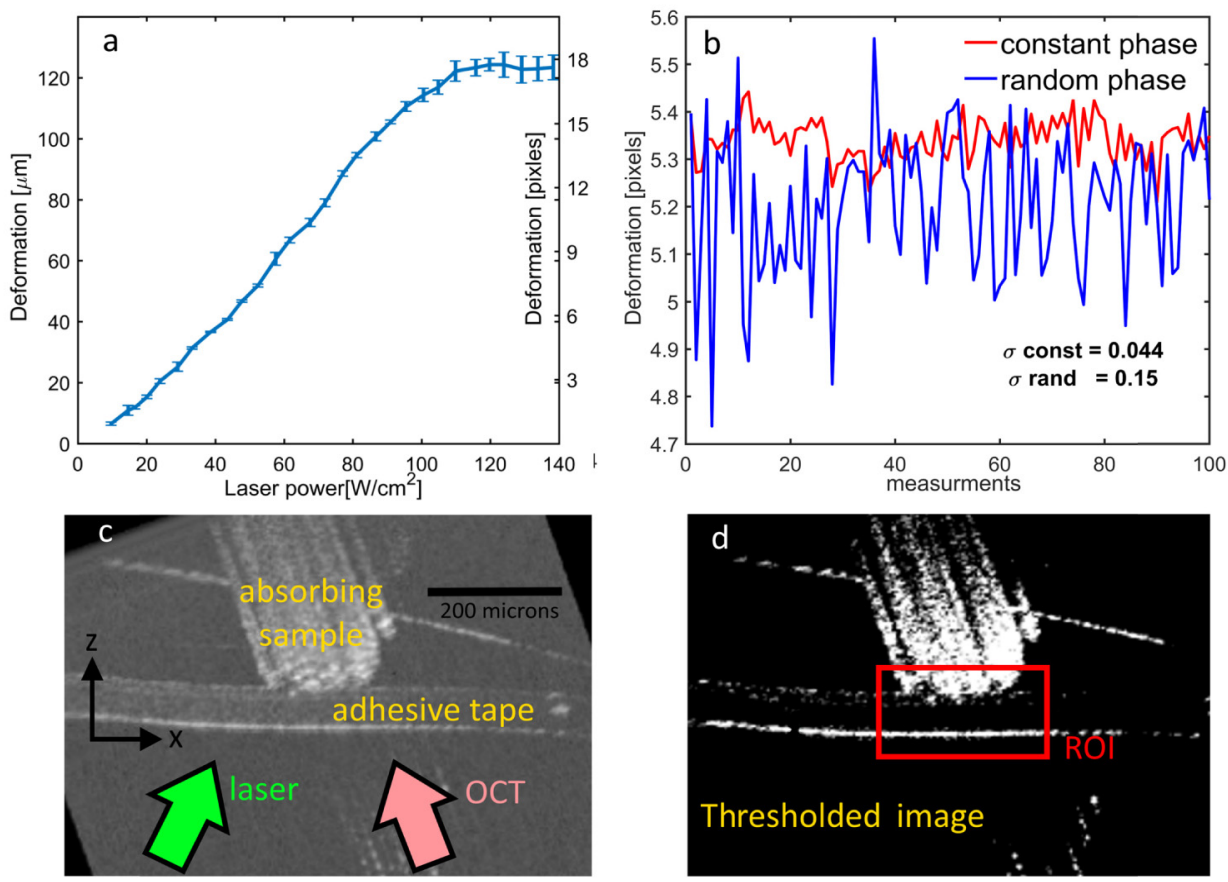

Fig. 3. System characterization results. a- Deformation of the sample as a function of green laser power. b- SNR characterization. Preliminary comparison of a series of random phase patterns with a constant phase pattern. c- Gray scale OCT image. d- Thresholded image and ROI for deformation detection (see Visualization 1).

We demonstrate the ability to enhance light focusing, in a selected focal area inside the sample, using the thermal expansion feedback. For this demonstration, the black polyester sewing thread sample faces the OCT at roughly $45^{\circ}$. The $514 \mathrm{~nm}$ laser passes through a diffuser, producing an intensity pattern on the sample. The adjusted diffuser configuration allows precise control over the experiment scattering properties. For high contrast speckles, the focusing objective was tuned slightly before the focal region on the diffuser. This illumination configuration corresponds to the results depicted in Figs. 4(a)-4(c). A mixture of scattered and ballistic photons was achieved by focusing the penetrating laser on the diffuser surface corresponding to the results depicted in Figs. 4(d)-4(f). Demonstration of both cases shows that any combination of the two scattering regimes can be controlled similarly.

Moreover, this configuration facilitates optical validation of the enhanced focused light, by removing the sample while keeping the diffuser in place, and directly imaging the speckle field. The wave-front optimization was implemented by a Genetic Algorithm (GA) [28]. Accordingly, SLM phase patterns are optimized based on the amplitude of the detected deformations, presented as distance in OCT pixels. The process starts with a set of random phase patterns and iteratively converges to an optimized phase mask by a natural selection that mimics biological evolution. Upon optimization, the calculated phase mask precompensates random scattering events inside the media, the deformations are enhanced, and an enhanced focal point is formed. The GA optimization process is depicted in Figs. 4(a) and 4(d), including the whole population at every iteration. Verification of the focusing was performed using a backside camera. The optimal phase mask was a combination of phase patterns, which has no particular observable trend, as shown in the insets of Fig. 4. 

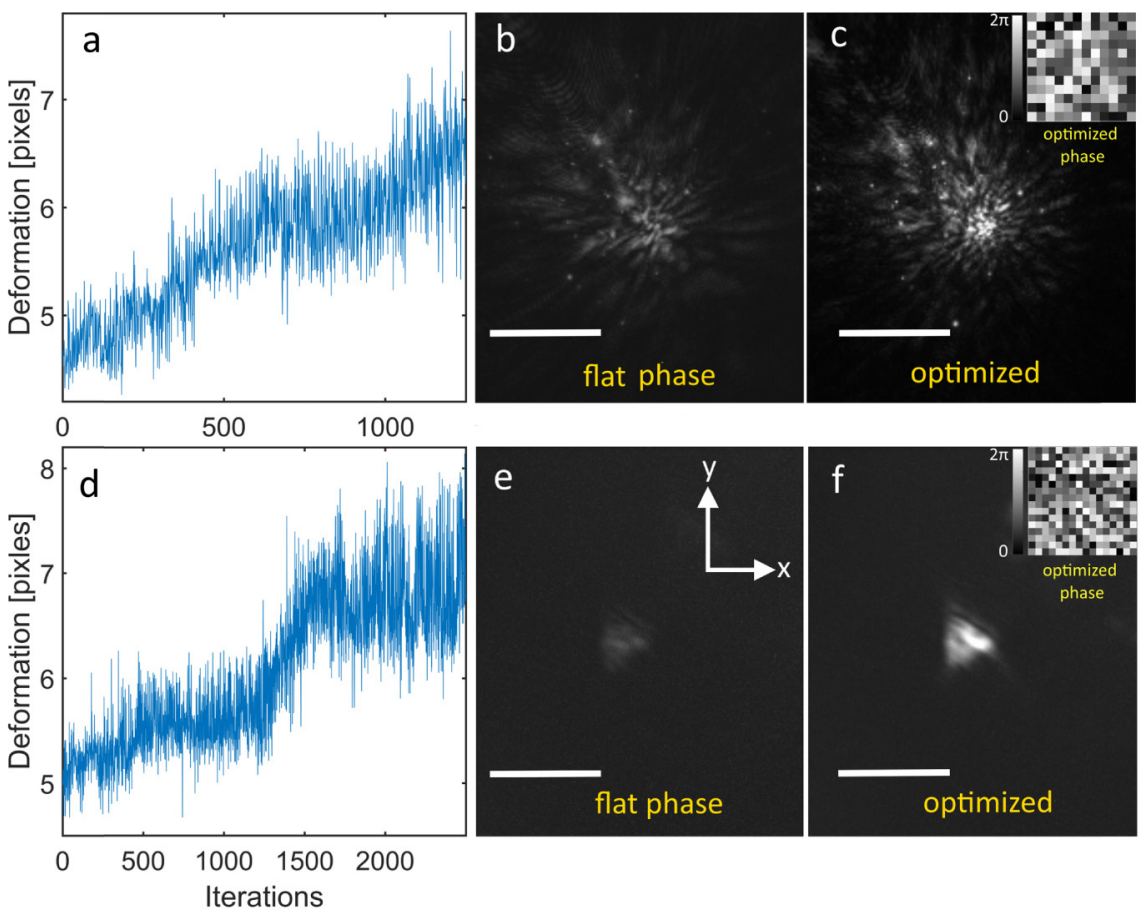

Fig. 4. Results of focusing through scattering media using thermal expansion feedback implemented with OCT. a- GA Optimization in the case of high contrast speckles. b- Backside camera image of scattered light without the sample and with a flat SLM phase. c- Backside image using the optimized phase pattern. d- GA Optimization in the case of ballistic and scattering mixture. e- Flat SLM phase. f- Optimized phase pattern. The top right inserts in c and f depict the optimized SLM phase patterns with 144 and 256 macro-pixels respectively. Scale bar is $1 \mathrm{~mm}$.

After the optimization, the sample was removed, and the speckle pattern was imaged using a backside camera, as shown in Figs. 4(b), 4(c), 4(e), and 4(f). The peak intensity to average background ratio, also known as enhancement $\eta$, was measured [5]. The experimental results recorded are, $\eta_{1}=2.05$, for the high contrast speckles, and $\eta_{2}=2.8$, for the predominantly ballistic light. The theoretical enhancement is proportional to the number of controlled degrees of freedom, $N_{S L M}$, and inversely proportional to the number of optical modes (speckle grains) whose intensity is enhanced, $N_{\text {modes }}$. In our case the number of enhanced speckles, as depicted in Fig. 4(c), is $N_{\text {modes }} \sim 15$. The expected intensity enhancement [13] is $\eta_{\text {expected }}=\alpha\left(\frac{N_{S L M}}{N_{\text {modes }}}\right) \sim 0.5\left(\frac{144}{15}\right)=5$, where $N_{S L M}$ corresponds to $12 \times 12$ SLM macro pixels. The factor $\alpha$ depends on the type of light modulation that is used, and on the intensity distribution over the SLM. The lower experimental optical enhancement was caused mainly by optical speckle decorrelation during the experiments, and noise in the measurements. The overall time of the optimization was several hours (limited by the OCT device acquisition speed of $\sim 5 \mathrm{sec}$ per image). As such, it is likely that mechanical drifts, reduced the speckle decorrelation time, and limited the total enhancement of the optimization. Note that the substrate and the scattering layers are characterized by low optical absorption and did not deform thermally, even under intense illumination (up to an order of magnitude higher fluence). Only the presence of an absorber created detectable thermal deformations, which confirmed that the focus spot, created by the GA optimization, resides in the absorber. 
Furthermore, the total transmission behind the sample was reduced after focusing due to the enhanced absorption.

\section{Discussion}

The sensitivity of TEF-WFS, and hence the ability to focus light efficiently, depends on the sensitivity of the deformation detection. Even with sophisticated motion detection algorithms [29], OCT imaging resolution and sensitivity remain the primary limitations. In traditional OCT, the depth resolution, typically on the order of micrometers, is defined by the coherence length, and the transverse resolution, typically also of the same order, depends on the minimum waist radius of the OCT beam [17]. Therefore, the detection of minute deformation changes with alternating SLM patterns is challenging (see Fig. 3(b)), and the applicability of the method is limited to highly absorptive features with relatively large thermal expansion coefficients. The method could be improved with use of phase-sensitive optical coherence tomography (PSOCT). The OCT phase information is highly sensitive to optical path changes and capable of measuring nanometric deformations [30,31]. It is worth noting that PSOCT has already been used for optical coherent elastography (OCE), which takes advantage of nanometric deformations to study and characterize tissue properties [32]. Employing PSOCT and deformation detection techniques used in OCE [33] for feedback, could considerably enhance the sensitivity of TEF-WFS and provide a better signal to control the wave-front. As a result, focusing inside scattering media would potentially be possible with fewer limitations on the thermal properties of the absorbing structure. Sufficient SNR for phase measurements in highly scattering media would be required for implementing PSOCT-based feedback since the phase signal decreases significantly with scattering.

The ballistic component $I_{B}$ of the total transmitted intensity decays exponentially across a scattering layer of thickness L [34], $I_{B}=I_{0} e^{-L / l_{s}}$, where $l_{s}$ is the scattering mean free path. Therefore, the SNR of OCT drops rapidly with multiple scattering events, reducing the image quality in depth beyond $1-2 \mathrm{~mm}$ using IR wavelengths. Visible wavelengths have much shorter $l_{s}$ and therefore penetrate less inside biological tissues [35]. In other words, longer wavelengths are characterized by lower scattering, $\mu_{s} \approx \frac{1}{l_{s}}$, both in Mie [36] and Rayleigh scattering regimes. Specific examples for scattering media in which the scattering of the OCT IR wavelength is much lower than scattering for the penetrating laser wavelength include human skin [37], the brain [38], and other biological tissues [39]. On top of the high penetrating wavelength in the IR range, TEF-WFS exploits coherence gating of ballistic photons. Both mechanisms enable control over visible highly scattered light within the penetration of the OCT. In a more realistic scenario, the same amount of scattering should have been placed on both the OCT, and penetrating laser optical paths. However, in order to increase the SNR of the OCT signal, we tested the performance with the diffuser only on the path of the penetrating laser. This setup is equivalent to a sample with low scattering for the OCT IR SLD and high scattering for the visible wavelength laser.

We selected a sample with high linear thermal expansion coefficients, resistant to high laser fluence and situated in such a geometry that produces high 3D thermal deformations and translations. We do not expect such level of deformation in tissues. However, in terms of thermal expansion, tissues are typically modeled as water, due to their high water content.

The thermal expansion of water $\left(\sim 240 \times 10^{-6}\left[\frac{\mathrm{m}}{\mathrm{mK}}\right]\right)$ is comparable to that of our samples ( $\left.130 \times 10^{-6}\left[\frac{\mathrm{m}}{\mathrm{mK}}\right]\right)$. Additionally, there have been several demonstrations of photo-thermal deformation measurements with OCT in real tissue and tissue phantoms [40-44]. Note that, in 
the experiments, the goal is always to optimize the wavefront below damage threshold and then create local energy deposition with possible targeted tissue damage at a selected ROI.

Conceptually, TEF-WFS is similar to focusing light using photoacoustic feedback [11]. In this method, light absorption creates acoustic waves which suffer minimal scattering, are detected by acoustic transducers, and then used as feedback. In our case, low frequency thermal deformations are recorded via OCT, enabling enhanced control and focusing of scattered light within the penetration range of the IR coherence gated imaging. Photoacoustic imaging is capable of deeper penetration depth, higher SNR and shorter measurement period compared to OCT. However, there are relative advantages of OCT based feedback: First, OCT feedback has optical resolution inside tissue, typically higher than the resolution of the photoacoustic feedback. Secondly, OCT is air coupled and does not require impedance matching such as photoacoustic imaging. This advantage might be relevant in applications such as ophthalmology where impedance matching complicates the clinical procedure. Lastly, OCT is a widespread imaging technology, commonly used in biomedicine, which could facilitate integration and adoption with wavefront shaping. As such, combining a wave-front shaping system with OCT clinical systems is a realistic, practical path that could enhance applications such as photodynamic therapy, which often require photons in the visible range tuned to specific chromophore absorption bands.

TEF-WFS enables improved performance by a combination of a high NA objective for the penetrating laser with low NA lens for the feedback OCT. In addition, the OCT arm enables on-line dynamic monitoring of the sample during all phases of energy deposition as well as precise selection of the focusing target inside complex tissue. The thermal deformations we measured are on the order of hundreds of milliseconds for CW lasers and are governed by steady state thermal equilibrium timescales in the material. Smaller deformations and pulsed lasers could have shorter characteristic time scales. Improved sensitivity of the OCT, e.g. through phase detection with a state-of-the-art swept source OCT system, would allow measuring much smaller deformations and significantly reduce the inherent measurement time. Real-time focusing in highly dynamic turbid tissue with speckle decorrelation times on the order of milliseconds would still be challenging. However, our method could be advantageous for more static scenarios such as dental applications.

\section{Conclusions}

In conclusion, we presented a new wave-front shaping method for focusing through scattering media: TEF-WFS, enabled by OCT. Proof of concept experiments demonstrate that laserinduced, reversible thermal expansions can be measured and used as a feedback mechanism for wave-front shaping optimization. Enhanced focusing of visible light is attained at depth ranges of the coherent gated IR feedback. Hence, the method has potential for a new biomedical focusing/imaging modality in which the penetrating laser focuses energy on selected features inside scattering tissue, while being monitored and optimized dynamically using an OCT system. Potential bio-medical applications include photodynamic therapy.

\section{Funding}

This work was supported by the National Science Foundation (award \#1611513).

\section{Acknowledgments}

We thank Christoph Meier for useful discussions. 